\title{
How comparative psychology can shed light on human evolution: Response to Beran et al.'s discussion of "Cognitive capacities for cooking in chimpanzees"
}

\author{
Alexandra G. Rosati $^{1}$ (D) $\cdot$ Felix Warneken ${ }^{2}$
}

Published online: 23 March 2016

(C) Psychonomic Society, Inc. 2016

\begin{abstract}
We recently reported a study (Warneken \& Rosati Proceedings of the Royal Society B, 282, 20150229, 2015) examining whether chimpanzees possess several cognitive capacities that are critical to engage in cooking. In a subsequent commentary, Beran, Hopper, de Waal, Sayers, and Brosnan Learning \& Behavior (2015) asserted that our paper has several flaws. Their commentary (1) critiques some aspects of our methodology and argues that our work does not constitute evidence that chimpanzees can actually cook; (2) claims that these results are old news, as previous work had already demonstrated that chimpanzees possess most or all of these capacities; and, finally, (3) argues that comparative psychological studies of chimpanzees cannot adequately address questions about human evolution, anyway. However, their critique of the premise of our study simply reiterates several points we made in the original paper. To quote ourselves: "As chimpanzees neither control fire nor cook food in their natural behavior, these experiments therefore focus not on whether chimpanzees can actually cook food, but rather whether they can apply their cognitive skills to novel problems that emulate cooking" (Warneken \& Rosati Proceedings of the Royal
\end{abstract}

Alexandra G. Rosati and Felix Warneken contributed equally to this work.

Alexandra G. Rosati

rosati@fas.harvard.edu

Felix Warneken

warneken@wjh.harvard.edu

1 Department of Human Evolutionary Biology, Harvard University, Cambridge, MA 02138, USA

2 Department of Psychology, Harvard University, Cambridge, MA 02138, USA
Society B, 282, 20150229, 2015, p. 2). Furthermore, the methodological issues they raise are standard points about psychological research with animals - many of which were addressed synthetically across our 9 experiments, or else are orthogonal to our claims. Finally, we argue that comparative studies of extant apes (and other nonhuman species) are a powerful and indispensable method for understanding human cognitive evolution.

Keywords Comparative cognition · Evolution . Chimpanzees $\cdot$ Cooking

\section{The rationale of our study}

The context of our study is a debate about the origins of cooking in human evolution. In particular, a recent hypothesis (Wrangham, 2009; Wrangham, Jones, Laden, Pilbeam, \& Conklin-Brittain, 1999) proposed that the shift to a cooked diet occurred fairly early and played a significant role in shaping the evolution of significant human traits, such as large brains. Testing this hypothesis therefore hinges on dating the emergence of cooking. Previous work has examined this question from the perspective of archaeological or fossil evidence. Yet, the archaeological record for hearths and burned material is fragile and may degrade, so there are disagreements concerning earliest evidence for the control of fire (e.g., Berna et al., 2012; Gowlett \& Wrangham, 2013; Roebroeks \& Villa, 2011). Moreover, fire can also be used for warmth, light, and protection (Bellomo, 1994; Burton, 2009), so even strong evidence for fire control might not necessarily reflect the emergence of cooking. Shifts toward a cooked diet might be reflected by morphological reductions in teeth or gut size (Organ, Nunn, Machanda, \& Wrangham, 2011; Wrangham, 2009), but this fossil evidence could also reflect increased 
consumption of other softer or higher quality foods (Aiello \& Wheeler, 1995; Teaford \& Ungar, 2000; Ungar, Grine, \& Teaford, 2006).

We therefore proposed that comparative psychological studies could provide a third window into the origins of cooking. As cooking behaviors require multiple cognitive skills (beyond the control of fire), studies of the psychological capacities in other species - especially chimpanzees, as one of our closest living relatives - can provide a valuable test case for how rapidly the control of fire may have led to the adoption of cooking. We used an analytical approach and broke cooking down into a set of constituent skills that we argued are necessary prerequisites for this behavior. We then systematically tested these skills across nine experiments that built in complexity while targeting different components. For example, our experiments initially confirmed that chimpanzees selected cooked food over a raw alternative when faced with a dichotomous choice (Experiments 1 and 5a: potatoes; Experiments 6a: carrots). Another study presented chimpanzees with a choice between one raw piece of food they could have immediately, or three pieces they could obtain only after a 1-minute delay; the critical comparison was how chimpanzees responded when this larger reward was raw versus cooked across conditions. We found that chimpanzees were more willing to wait for the delayed food when it was cooked than raw, indicating that they would pay some additional temporal cost to acquire it (Experiment 2). The majority of our tasks (Experiments 3-9) then involved two novel devices: a cooking device that appeared to transform raw food into cooked food when food was placed inside and the device was shaken, and a control device that could be manipulated in the same way but did not transform raw food. We used these devices — rather than "real" cooking, such as with a fire- not only for pragmatic safety reasons but also to test chimpanzees on a novel problem emulating cooking that they could never have seen before.

Using this basic setup, we probed how chimpanzees understood these devices. First, we found that chimpanzees preferred the cooking device over the control device after seeing both baited with raw food and manipulated, but not yet opened to reveal their contents (Experiments 3 and 5b). Second, when directly given raw food, chimpanzees chose to actively place it in the cooking device in order to acquire cooked food rather than eat the food already in their possession or place it in the control device that did not alter it (Experiments 4 and 5c). Third, chimpanzees flexibly modulated what they put in this device: they placed both familiar and novel raw foods inside (carrots, which the chimpanzees had never observed with the devices) rather than placing them in the control device, but directly ate precooked foods (cooked potato) that did not need to be transformed in this way (Experiment 6b). Fourth, when given both a slice of raw potato and a visually similar but inedible item (a wood chip) at the same time, they selectively chose to place the edible item inside the cooking device, but did not attempt to place the wood chip or both options inside - even though they might have thought that this would have increased their total food rewards (Experiment 7). That is, chimpanzees did not merely think something superior came out of the cooking device whenever they placed something inside, as they refrained from putting in nonfood items. Fifth, they exhibited additional self-control capacities by transporting raw food across a room, paying some additional energetic and temporal costs to place it in the cooking device (Experiment 8). Finally, to a more limited degree they saved raw food for 3 minutes in anticipation of future opportunities to place it in the cooking device (which was not present at the time they chose to save the food), but ate the entire allotment of raw food in a control condition where there were no such opportunities to "cook" the food (Experiment 9).

Based on this whole sequence of experiments, we argued that chimpanzees show several of the psychological capacities that are necessary to cook food: motivation or desire to pursue cooked food; patience to wait temporal delays to acquire cooked foods as well as pay some additional energetic cost to transport food so it would be cooked; the self-control to give up food in one's hand to have it be cooked; a basic causal comprehension of how these devices transformed raw foods after little experience; and, finally, the ability to save raw food for future cooking opportunities. We then reflected on additional technological, social, and ecological factors that may have been necessary for cooking behaviors to actually emerge in the hominin lineage. In particular, we highlighted the need for control of fire; increased interindividual tolerance due to the risk of theft posed by the cooking process given that many apes are quite competitive for food; and a shift to a diet including more starchy tubers (which are profoundly transformed by cooking, unlike the fruit staples of chimpanzee diets). That is to say, while we proposed and tested for several psychological skills that we think are necessary prerequisites for cooking, we also indicated that this list was unlikely to be exhaustive given that chimpanzees do not in fact cook food.

\section{Critique 1: are these methodologies appropriate?}

Many aspects of Beran et al.'s commentary focus on whether our methods could assess whether chimpanzees possess "conceptual knowledge" of cooking (Beran et al., 2015, p. 1). For example, their commentary analogizes our cooking devices to a microwave: chimpanzees may understand that they can put a raw slice of potato in and get a cooked slice out, but not anything deeper about the underlying physical transformation. Beran et al. contest our claims about chimpanzees' competence because our setup did not require "any knowledge about why a better food emerged" (Beran et al., 2015, p. 2), or that shorter cooking periods result in undercooked food and long cooking periods may burn the potatoes (p. 3). We agree that it 
may very well be the case that chimpanzees treated our cooking device in the way humans use a microwave but disagree that this proposal affects our main conclusions. At the heart of this debate is what abilities an organism (human or otherwise) actually has to have in order to cook foods. To our knowledge, there is no overarching theory that specifies the necessary and sufficient behavioral skills for organisms to cook. Therefore, our approach was to suggest and test for basic cognitive and behavioral skills whose absence would preclude cooking behaviors. For example, an individual would need to be willing to wait some temporal duration in order to cook food, as cooking inherently takes some time-without necessarily understanding thermodynamics. Similarly, an individual would have to understand that his or her cooking behavior turns raw food into cooked food (whether this is by placing food into a fire or inside a microwave), without necessarily knowing how or why this happens chemically. We therefore argued that our results suggest that chimpanzees had "a practical understanding of this basic cooking transformation after minimal experience" (Warneken \& Rosati, 2015, p. 8, emphasis added). Thus, the premise of our work was not that chimpanzees would have evolved "conceptual knowledge" of cooking - which would be a rather odd claim given that chimpanzees do not naturally cook. Rather, we argued that many of the skills needed for cooking are domain-general capacities, likely used in other contexts such as foraging, which could be applied to the problems posed by cooking. Thus, we agree that in effect we presented the chimpanzees with an artificial microwave paradigm, but believe that this situation actually captures many critical behavioral dimensions of cooking.

Another major point in Beran et al.'s commentary is that many of our results might stem from of associative learning. Specifically, they propose that "chimpanzees associated, through experience and learning, one container with the more-preferred cooked potato, and one with the lesspreferred raw potato, and they chose the container associated with the more-preferred food" (Beran et al., 2015, p. 2). We agree that it is obvious that some form of learning must account for the chimpanzees' responses across our tasks, as we designed the studies to involve an entirely new problem vis-àvis the novel cooking and control devices. The main question at stake is: Does it challenge our claim that chimpanzees' possess critical capacities for cooking if the chimpanzees' responses stemmed from associative learning mechanisms? We think it does not. By whatever means, chimpanzees have some psychological machinery that is sufficiently complex for them to quickly learn cooking-relevant skills. So, even if we were wrong in the specific inferential capacities we attribute to chimpanzees, this alternative does not affect our main conclusion concerning their ability to solve several problems that emulate cooking.
Nonetheless, Beran and colleagues (2015) think our specific claims about the underlying psychology are wrong and present their alternative as a deflationary account. Yet, we find the commentary's particular associative learning account to be implausible. Their proposed association of the cooking device with preferred cooked potatoes might at best explain why the chimpanzees chose to retrieve food from the cooking device over the control device (Experiment 3). But, it is unclear how this could account for the fact that chimpanzees also opted to actively place their own food inside the cooking device (Experiments 4-9), or even save food for the future when no cooking device was even present at the time (Experiment 9). These responses require the self-control to give up food in their hand - not just to associate the cooking device with preferred food - as well as additional inferential steps as to the outcomes of their actions. Moreover, this scenario is not compatible with the fact that the chimpanzees learned these responses rapidly and then flexibly deployed their new knowledge. For example, the chimpanzees did not blindly place any item in the cooking device under the assumption that a good food item would pop out: they selectively placed familiar and novel raw foods inside but chose to eat precooked food and ignore inedible items (Experiments $6 \mathrm{~b}$ and 7). In total, our results are not compatible with the particular associative learning account described by Beran and colleagues.

Other methodological critiques of specific experiments in our paper seem unfounded. For example, the commentary argues that it is difficult for nonhuman primates to inhibit pointing to larger amounts of food in their critique of Experiment 2 on temporal discounting. However, this study contrasted two conditions that both involved a choice between a smaller, immediate reward (raw in both conditions) and a larger reward that required a 1-min wait (raw or cooked, across conditions). Given that this potential "pointing" issue would then apply to both conditions, it is unclear how it can account for the chimpanzees' differential willingness to wait for cooked foods. Moreover, other species do show preferences for the smaller, immediate reward in intertemporal choice tasks when the larger reward is delayed for even short periods, on the order of 10-20 s (Stevens \& Stephens, 2008). This includes cotton-top tamarins (Saguinus oedipus), marmosets (Callithrix jacchus), and several lemur species tested in quite similar contexts with visible food rewards (Stevens, Hallinan, \& Hauser, 2005; Stevens \& Muhlhoff, 2012). Given that great apes show much more robust inhibitory control over prepotent reaching responses compared to other primates (see Maclean et al., 2014, for a large cross-species comparison), it is difficult to see how this pointing explanation could account for this complete set of results. Similarly, Beran et al. write that chimpanzees' placing of raw food items into the cooking device might reflect trading behavior, similar to previous studies (e.g., Dufour, Pelé, Sterck, \& Thierry, 2007). Yet, as we pointed out in our original paper, such previous studies involved 
extensive initial shaping of this trading behavior, and the chimpanzees in our study did not experience such trainingnor did they have other relevant learning experiences, such as in the use of tokens. Moreover, our experiments testing whether this behavior transfers to other food items but not nonfood items directly addressed this trading hypothesis, as discussed below.

The commentary also raises the specter of potential social cuing in our studies. This is a common issue in comparative research involving human experimenters, and we took the standard approach for dealing with it: The experimenter looked down or along the table's midline to avoid inadvertent cues when the ape was responding (Warneken \& Rosati, 2015, p. 3). Yet, in general, over two decades of research has shown that apes are not very successful at using even highly salient human social cues to locate hidden food-including directed gaze, pointing, or placing physical markers on containers (see Hare, 2011; Tomasello, Call, \& Hare, 2003a, for reviews). Chimpanzees can certainly use social cues in some contexts more successfully than others: they are more successful at interpreting a human competitor reaching for a piece of hidden food compared to a human cooperatively pointing at its location (Hare \& Tomasello, 2004) and can learn to use humans' pointing gestures to identify which tube to place an object in following extensive training with this trading procedure (Hopkins, Russell, McIntyre, \& Leavens, 2013). Overall, however, they have at best a fragile ability to use even clearly visible and intentional human social cues in the absence of training (see Rosati, Santos, \& Hare, 2010, for a review). Although it is of course important to be vigilant to the possibility inadvertent social cuing, there is no actual evidence of chimpanzees using subtle cues that the human actor did not actually intend to produce. Most importantly from our perspective, evidence for social-cue use comes primarily from situations where animals have to make a choice by reaching or pointing to one of two options, but we only used this method for the first three experiments in our paper. There is no evidence that a human cue could cause an animal to spontaneously give up food they already possess, the primary method we used in Experiments 4 through 9, involving the cooking device. In Experiment 9 there was not even a human around to produce such an inadvertent social cue, as that study examined the chimpanzees' ability to save the food for the future in the absence of concurrent cues that cooking was possible, such as the experimenter or the devices.

Finally, we must point out that Beran and colleagues' approach in their commentary is to suggest alternative explanations for each individual experiment in our paper rather than consider the entire weight of the evidence. It is certainly important to identify what questions individual studies leave unanswered. However, it is curious that several of their concerns simply restate comments we made in our original paper, without considering how subsequent experiments were explicitly designed to address these outstanding questions. For example, we first suggested that chimpanzees might have learned to trade raw potato for cooked potato (in Experiments 4 and 5), but we then went on to test whether this was the case (in Experiments 6 and 7). In fact, chimpanzee neither placed only raw potato into the cooking device (a narrow "trading" interpretation) nor optimistically placed any item into it (a broader interpretation). Rather, they flexibly and selectively shifted their behavior to place both familiar and novel raw items but not precooked or inedible items. We think this issue speaks to how converging evidence can be used to evaluate alternative explanations for the mechanisms underlying behavior. Indeed, similar discussions about how ad hoc alternatives for specific results can be ruled out have occurred for other domains in comparative psychology (e.g., see Call \& Tomasello, 2008; Povinelli \& Vonk, 2003; Tomasello et al., 2003a; Tomasello, Call, \& Hare, 2003b, for a debate about chimpanzees' ability to engage in visual perspective-taking, where interpretations based on narrow behavior-reading rules might possibly account for some results but do not apply to other findings). Of course, the results from any one experiment may have multiple interpretations - the key is whether those interpretations hold up across all the evidence. In this case, it does not.

\section{Critique 2: this is all old news}

The second major point made in Beran and colleagues' commentary is that our findings are not actually novel. They argue that previous studies have already demonstrated that chimpanzees show causal reasoning, can delay gratification, or plan for the future. We think that this comment is problematic in at least two ways. The first concerns the goal of (comparative) psychological research. In our opinion, we should not just aim to check off whether a certain cognitive ability is present or absent in a particular species - it is also critical to assess how and when these cognitive abilities are actually used across different contexts. Even if component abilities are present in a certain organism (in that they show them in some situations), it does not necessarily follow that they apply these skills to all situations or can integrate them with other relevant skills to perform a complex behavior. In fact, several theories from cognitive development (Carey, 2004), cognitive neuroscience (Buckner \& Krienen, 2013), and comparative cognition (Shettleworth, 2012) assert that unique components of human cognition may in a large part be due to novel connections between different cognitive abilities. That is, nonhumans might have many or all the relevant constituent parts seen in humans but cannot integrate these skills in the way necessary to give rise to novel, human-specific behaviors (e.g., Spelke \& Kinzler, 2007; Wang \& Spelke, 2002). 
The second problem of this critique pertains to the goals and claims of our study in particular. Our paper acknowledged that many of our target cognitive skills have been the focus of (sometimes extensive) research in comparative psychology. Specifically, we emphasized that cognitive abilities such as temporal discounting, self-control, causal reasoning, and future planning may be domain-general capacities that chimpanzees can apply across different contexts: "the cognitive skills we explore are not necessarily specific to [cooking]" (Warneken \& Rosati, 2015, p. 2). For example, many researchers have examined temporal discounting (see Stevens $\&$ Stephens, 2008, for a review) or future planning in primates, birds, and other species (see Clayton, Bussey, \& Dickinson, 2003; Mulcahy \& Call, 2006; Osvath \& MartinOrdas, 2014). Yet, we would be quite surprised to learn there was already consensus that chimpanzees (or any other nonhuman) can use them for cooking in particular. The fact that many of our target cognitive skills are of great interest to comparative psychologists does not mean that previous work made any connection to cooking. As a matter of fact, these prior studies did not even raise the question of what abilities may underlie cooking behaviors.

To our knowledge, the only previous study specifically targeting the psychological skills that support cooking behaviors comes from a paper by Wobber, Hare, and Wrangham (2008), showing that all four great ape species preferred a variety of food when cooked. This corresponded to the question we asked in our first experiment, and we framed that experiment as a replication of this earlier study. Thus, our paper acknowledged our debt to that work but also clearly built on this foundation in subsequent studies (e.g., Experiments 2-9). Indeed, some questions addressed in our work-such as whether chimpanzees can save food (or anything) for the future-are currently under contention (Suddendorf \& Corballis, 2007). In sum, with the exception of Wobber and colleagues' (2008) study focusing on food preferences, the previous psychological work had not asked the question whether chimpanzees (or any other animal) can utilize their existing skills in a context that emulates cooking.

Along the same lines, our focus on chimpanzees in this particular study does not mean that only chimpanzees possess this suite of skills. We focused on chimpanzees because they are one of our closest living relatives and show many sophisticated capacities for future-oriented cognition in other situations (Osvath \& Martin-Ordas, 2014). But as already detailed, we argued that many of the skills needed to cook food are domain-general capacities that are not specific to cooking. As such, some of the future-oriented skills we explored might also be present in other species as well. For example, these capacities might be shared by common descent with the other living great apes — or be present in corvids, who exhibit many complex future-oriented behaviors, through evolutionary convergence (Emery \& Clayton, 2004). The preference for cooked foods likely reflects a basic sensitivity to qualities such as sugar or glutamate content and appears to be widely shared (see Carmody, Weintraub, \& Wrangham, 2011; Wobber et al., 2008; Wrangham, 2009, for discussion). This sort of evidence does not contradict our claims - rather, it provides even stronger phylogenetic support for the hypothesis that early hominins also had these capacities.

\section{Critique 3: chimpanzees are not a good model for human evolution}

The final-and in our opinion most provocative - set of claims in Beran and colleagues' commentary concerns the relevance of chimpanzee cognition for understanding the emergence of cooking. Beran and colleagues repeatedly express puzzlement as to how chimpanzees, who do not naturally cook, could possess any cognitive capacities used for the purpose of cooking. We agree there is no reason that chimpanzees would have evolved special psychological abilities specific to cooking, but this is not what we argued in our paper. As detailed previously, our study focused on a set of cognitive prerequisites that are necessary to engage in cooking behaviors, not "conceptual knowledge" of cooking itself, let alone some domain-specific cooking ability. We proposed that many of the skills needed for cooking are domain-general capacities that are likely utilized in other contexts such as foraging. From this perspective, it does not seem unreasonable that chimpanzees might exhibit some of these skills that happen to be essential prerequisites for cooking.

Furthermore, it seems obvious that the first creature who placed food in a fire for the first time - whether that creature was Homo erectus or Homo sapiens - could not yet have evolved capacities specific to cooking. Importantly, this chicken-or-egg problem in understanding the sequential evolution of important traits is not a problem specific to cooking. In fact, it stems back to the older concept of preadaptation and its more recent formation of exaptation (Gould \& Vrba, 1982): the idea that traits that evolved for one function can be later coopted for a new one. When considered from the perspective of cooking, we would therefore suggest that the set of cognitive traits we examined most likely serve other behavioral functions in nonhumans and were then co-opted for novel cooking behaviors sometime in the human lineage. Indeed, in our paper we argued that "opportunistic use of natural firesunderpinned by the types of cognitive capacities examined in the current studies - may have played an important role in bootstrapping the emergence of more complex cooking behaviours that required the active control and maintenance of fire" (Warneken \& Rosati, 2015, p. 8) - a point reiterated by Beran et al in their commentary. Thus, an important future question is whether such cognitive capacities were further refined in human evolution to specifically reflect new 
cognitive challenges posed by the control of fire and dietary dependence on cooked foods.

Finally, the commentary suggests that our studies are limited by the fact that chimpanzees are "not ideal surrogates" (Beran et al., 2015, p. 4) for the last common ancestor of human and other apes. In particular, Beran et al. argue that recent fossil evidence from Ardipithecus (Lovejoy, 2009) shows that the last common ancestor was not chimpanzeelike (or more generally African-ape-like) as previously supposed. Lovejoy and colleagues explicitly argue that Ardipithecus invalidates models of human origins based on living African apes (see also Sayers \& Lovejoy, 2008; Sayers, Raghanti, \& Lovejoy, 2012, for extended critiques of evolutionary models based on chimpanzees). For example, Sayers and Lovejoy (2008) stated that "chimpanzee data have been consistently misapplied in discussions of human origins and that attempts to account for the differentiation of hominids from great apes based on a strict Pan troglodytes model cannot succeed" (p. 87). This view would suggest that comparative studies of behavior and cognition in chimpanzees and other living apes are not of any special importance for understanding human evolution. We have serious concerns with this conclusion, and we are not alone. This claim that an African ape model for the last common ancestor is inaccurate has produced a flurry of critiques from evolutionary scientists, both on morphological grounds (e.g., Lieberman, 2013; Wood \& Harrison, 2011) as well as for the behavioral implications of this claim (McGrew, 2010; Stanford, 2012). In terms of cognition specifically, what could replace great apes as a better model for scientists interested in testing psychological hypotheses about human evolution? Beran and colleagues argue that, for cooking at least, the focus should be on Homo erectus. If we had access to a time machine and could conduct psychology experiments with extinct hominins, that would undoubtedly be the best option. In the meantime, however, we would assert that psychological comparisons of living nonhumans - and especially our closest living relatives, such as chimpanzees-represent the best bet to understand the evolutionary origins of human cognition.

\section{References}

Aiello, L. C., \& Wheeler, P. (1995). The expensive tissue hypothesis: The brain and the digestive system in human and primate evolution. Current Anthropology, 36, 199-221.

Bellomo, R. V. (1994). Methods of determining early hominid behavioral activities associated with the controlled use of fire at FXJJ-20 main, Koobi-Fora, Kenya. Journal of Human Evolution, 27, 173-195.
Beran, M. J., Hopper, L. M., de Waal, F. B. M., Sayers, K., \& Brosnan, S. F. (2015). Chimpanzee food preferences, associative learning, and the origins of cooking. Learning \& Behavior. doi:10.3758/s13420015-0206-x

Berna, F., Goldberg, P., Horwitz, L. K., Brink, J., Holt, S., Bamford, M., \& Chazan, M. (2012). Microstratigraphic evidence of in situ fire in the Acheulean strata of Wonderwerk Cave, Northern Cape province, South Africa. Proceedings of the National Academy of Sciences of the United States of America, 109, E1215-E1220.

Buckner, R. L., \& Krienen, F. M. (2013). The evolution of distributed association networks in the human brain. Trends in Cognitive Science, 17, 648-665.

Burton, F. (2009). Fire: The spark that ignited human evolution. Albuquerque: University of New Mexico Press.

Call, J., \& Tomasello, M. (2008). Does the chimpanzee have a theory of mind? 30 years later. Trends in Cognitive Sciences, 12(5), 187-192.

Carey, S. (2004). Bootstrapping \& the origin of concepts. Daedalus, 133, 59-68.

Carmody, R. N., Weintraub, G. S., \& Wrangham, R. W. (2011). Energetic consequences of thermal and nonthermal food processing. Proceedings of the National Academy of Sciences of the United States of America, 108, 19199-19203.

Clayton, N. S., Bussey, T. J., \& Dickinson, A. (2003). Can animals recall the past and plan for the future? Nature Neuroscience, 4, 685-691.

Dufour, V., Pelé, M., Sterck, E. H. M., \& Thierry, B. (2007). Chimpanzee (Pan troglodytes) anticipation of food return: Coping with waiting time in an exchange task. Journal of Comparative Psychology, 121, $145-155$.

Emery, N. J., \& Clayton, N. S. (2004). The mentality of crows: Convergent evolution of intelligence in corvids and apes. Science, 306, 1903-1907.

Gould, S. J., \& Vrba, E. (1982). Exaptation-A missing term in the science of form. Paleobiology, 8, 4-15.

Gowlett, J. A., \& Wrangham, R. W. (2013). Earliest fire in Africa: Towards the convergence of archaeological evidence and the cooking hypothesis. Azania: Archaeological Research in Africa, $48,5-30$.

Hare, B. (2011). From hominoid to hominid mind: What changed and why? Annual Review of Anthropology, 40, 293-309.

Hare, B., \& Tomasello, M. (2004). Chimpanzees are more skillful in competitive than cooperative cognitive tasks. Animal Behaviour, $68,571-581$

Hopkins, W. D., Russell, J., McIntyre, J., \& Leavens, D. A. (2013). Are chimpanzees really so poor at understanding imperative pointing? Some new data and an alternative view of canine and ape social cognition. PLoS One, 8, e79338.

Lieberman, D. E. (2013). The story of the human body: Evolution, health, and disease. New York, NY: Pantheon.

Lovejoy, C. O. (2009). Reexamining human origins in light of Ardipithecus ramidus. Science, 326, 74e71-74e78.

Maclean, E. L., Hare, B., Nunn, C. L., Addessi, E., Amici, F., Anderson, R. C., . . . Zhao, Y. (2014). The evolution of self-control. Proceedings from the National Academy of Sciences, 111(20), E2140-E2148.

McGrew, W. C. (2010). In search of the last common ancestor: New findings on wild chimpanzees. Philosophical Transactions of the Royal Society B, 365, 3267-3276.

Mulcahy, N., \& Call, J. (2006). Apes save tools for future use. Science, 312, 1038-1040.

Organ, C., Nunn, C. L., Machanda, Z., \& Wrangham, R. W. (2011). Phylogenetic rate shifts in feeding time during the evolution of Homo. Proceedings of the National Academy of Sciences of the United States of America, 108(35), 14555-14559.

Osvath, M., \& Martin-Ordas, G. (2014). The future of future-oriented cognition in non-humans: Theory and the empirical case of the great 
apes. Philosophical Transactions of the Royal Society B, 369, 20130486. doi:10.1098/rstb.2013.0486

Povinelli, D. J., \& Vonk, J. (2003). Chimpanzee minds: Suspiciously human? Trends in Cognitive Sciences, 7, 157.

Roebroeks, W., \& Villa, P. (2011). On the earliest evidence for habitual use of fire in Europe. Proceedings of the National Academy of Sciences of the United States of America, 108(13), 5209-5214.

Rosati, A. G., Santos, L. R., \& Hare, B. (2010). Primate social cognition: Thirty years after Premack and Woodruff. In M. L. Platt \& A. A. Ghazanfar (Eds.), Primate neuroethology (pp. 117-143). Oxford, England: Oxford University Press.

Sayers, K., \& Lovejoy, C. O. (2008). The chimpanzee has no clothes: A critical examination of Pan troglodytes in models of human evolution. Current Anthropology, 49, 87-114.

Sayers, K., Raghanti, M. A., \& Lovejoy, C. O. (2012). Human evolution an the chimpanzee referential doctrine. Annual Review of Anthropology, 41, 119-138.

Shettleworth, S. J. (2012). Modularity, comparative cognition and human uniqueness. Philosophical Transactions of the Royal Society B, 367, 2794-2802.

Spelke, E. S., \& Kinzler, K. D. (2007). Core knowledge. Developmental Science, 10, 89-96.

Stanford, C. B. (2012). Chimpanzees and the behavior of Ardipithecus ramidus. Annual Review of Anthropology, 41, 139-149.

Stevens, J. R., Hallinan, E. V., \& Hauser, M. D. (2005). The ecology and evolution of patience in two New World monkeys. Biology Letters, $1,223-226$

Stevens, J. R., \& Muhlhoff, N. (2012). Intertemporal choice in lemurs. Behavioural Processes, 89, 121-127.
Stevens, J. R., \& Stephens, D. W. (2008). Patience. Current Biology, 18, R11-R12.

Suddendorf, T., \& Corballis, M. C. (2007). The evolution of foresight: What is mental time travel, and is it unique to humans? Behavioral and Brain Sciences, 30, 299-351.

Teaford, M. F., \& Ungar, P. S. (2000). Diet and the evolution of the earliest human ancestors. Proceedings of the National Academy of Sciences of the United States of America, 97(25), 13506-13511.

Tomasello, M., Call, J., \& Hare, B. (2003a). Chimpanzees understand psychological states-The question is which ones and to what extent. Trends in Cognitive Sciences, 7(4), 153-156.

Tomasello, M., Call, J., \& Hare, B. (2003b). Chimpanzees versus humans: It's not that simple. Trends in Cognitive Science, 7, 239-240.

Ungar, P. S., Grine, F. E., \& Teaford, M. F. (2006). Diet in early Homo: A review of the evidence and a new model of adaptive versatility. Annual Review of Anthropology, 35, 209-228.

Wang, R. F., \& Spelke, E. S. (2002). Human spatial representation: Insights from animals. Trends in Cognitive Science, 6, 376-382.

Warneken, W., \& Rosati, A. G. (2015). Cognitive capacities for cooking in chimpanzees. Proceedings of the Royal Society B, 282, 20150229. doi:10.1098/rspb.20150229

Wobber, V., Hare, B., \& Wrangham, R. (2008). Great apes prefer cooked food. Journal of Human Evolution, 55, 340-348.

Wood, B., \& Harrison, T. (2011). The evolutionary context of the first hominins. Nature, 470, 347-352.

Wrangham, R. (2009). Catching fire: How cooking made us human. New York, NY: Basic Books.

Wrangham, R. W., Jones, J. H., Laden, G., Pilbeam, D., \& ConklinBrittain, N. (1999). The raw and the stolen: Cooking and the ecology of human origins. Current Anthropology, 40, 567-594. 